Theorems analogous to Newton's Theorems on Symmetric Functions, with Elementary Proofs.

By Dr R. F. Muirhead.

(Read 13th March 1914. Received 81st March 1914).

In his article in No. 15 of Mathematical Notes, Dr Dougall has shown how by introducing series with $n$ terms and a remainder, instead of infinite series, some well-known proofs of Newton's Theorems and others can be rendered more elementary in character. Once the idea has been suggested, it is of course easy to find further applications for it.

In what follows I propose to give some formulae which are closely analogous to Newton's though not so well known, and to furnish them with proofs in which Dr Dougall's idea is further applied.

\title{
Notation :-
}

I use $s_{1}, s_{2}, \ldots s_{r} \ldots$ for the sums of the $1^{\text {st }}, 2^{\text {nd }}, \ldots r^{\text {th }}$ powers of $n$ letters $\alpha, \beta, \gamma \ldots \lambda$; and $p_{1}, p_{2}, \ldots p_{r} \ldots p_{n}$ for the elementary symmetric functions of these letters; and $\mathrm{H}_{1}, \mathrm{H}_{2}, \ldots \mathrm{H}_{r} \ldots$ for the complete sums of homogenous products of the letters, of degrees $1,2, \ldots r, \ldots$ Thus $p_{r}$ stands for $\Sigma(\alpha \beta \ldots$ to $r$ factors) and denotes what is denoted by $(-1)^{r} p_{r}$ in Dr Dougall's article.

The following Lemmas will be proved:-

(i) $\Pi(1-\alpha x) \cdot\left\{1+\mathrm{H}_{1} x+\mathrm{H}_{2} x^{2}+\ldots+\mathrm{H}_{m} x^{m}\right\}=1+x^{m+1} \mathbf{R}_{m}$

$$
\Sigma \frac{\Pi(1-\alpha x)}{1-\alpha x}=n-(n-1) p_{1} x+(n-2) p_{2} x^{2} \ldots+p_{n-1}(-x)^{n-1}
$$

$$
\begin{aligned}
& \Sigma\left\{\left(1+2 \alpha x+3 \alpha^{2} x^{2}+. .+(m+1) \alpha^{m} x^{m}\right)\left(1+\beta x+\beta^{2} x^{2}+. .+\beta^{m} x^{m}\right)\right. \\
& \left.\ldots\left(1+\lambda x+\ldots+\lambda^{m} x^{m}\right)\right\} \\
& =n+(n+1) \mathrm{H}_{2} x+(n+2) \mathrm{H}_{2} x^{2}+\ldots+(n+m) \mathbf{H}_{m} x^{m}+x^{m+1} \mathbf{R}_{m} \\
& \text { (iv) } \Pi(1-\alpha x) \cdot \Sigma\left\{\left(1+2 \alpha x+3 \alpha^{2} x^{2}+\ldots+(m+1) \alpha^{m} x^{m}\right)\right. \\
& \begin{aligned}
(1+\beta x & \left.\left.+\ldots+\beta^{m} x^{m}\right) \ldots\left(1+\lambda x+\ldots+\lambda^{m} x^{m}\right)\right\} \\
& =n+s_{1} x+s_{2} x^{2}+\ldots+s_{m} x^{m}+x^{m+1} R_{m},
\end{aligned}
\end{aligned}
$$


where in each case the product-symbol $I I$ and the summation symbol $\Sigma$ extend over $n$ values of the typical factor or term, in which each of the $n$ letters $\alpha, \beta \ldots \lambda$ is in succession substituted for $\alpha$. $\mathrm{R}_{m}$ denotes in each case a polynomial in $x$ with a finite number of terms; and $m$ is any positive integer.

The formulae, analogous to those of Newton, and deduced from these Lemmas are as follows :-

I. $\mathrm{H}_{1}-p_{1}=0$

$$
\mathrm{H}_{2}-\mathrm{H}_{1} p_{1}+p_{2}=0
$$

$$
\mathrm{H}_{r}-\mathrm{H}_{r-1} p_{1}+\mathrm{H}_{r-2} p_{2} \ldots-(-1)^{r} \mathrm{H}_{1} p_{r-1}+(-1)^{r} p_{r}=0 .
$$

II. $\mathrm{H}_{1} p_{1}-2 p_{2}=\mathrm{s}_{2}$

$$
\mathrm{H}_{2} p_{1}-2 \mathrm{H}_{1} p_{2}+3 p_{3}=s_{3}
$$$$
\mathrm{H}_{r-1} p_{1}-2 \mathrm{H}_{r-2} p_{2}+\ldots+(-1)^{r}(r-1) \mathrm{H}_{1} p_{r-1}-(-1)^{r} r p_{r}=s_{r} .
$$

III. $s_{r}+s_{r-1} \mathrm{H}_{1}+s_{r-2} \mathrm{H}_{2}+\ldots+s_{1} \mathrm{H}_{r-1}=r \mathrm{H}_{r}$ and for comparison, we may write down Newton's formulae with the same notation, viz. :-

IV. $s_{1}-p_{1}=0$

$$
\begin{aligned}
& \boldsymbol{s}_{2}-\boldsymbol{s}_{1} p_{1}+2 p_{2}=0 \\
& \cdots \ldots \ldots \ldots \ldots \ldots \ldots \ldots \ldots \ldots \ldots \ldots \ldots \\
& \boldsymbol{s}_{r}-\boldsymbol{s}_{r-1} p_{1}+s_{r-2} p_{2}-\ldots-(-1)^{r} s_{1} p_{r-1}+(-1)^{r} p_{r}=0 .
\end{aligned}
$$

In all these formulae it is to be noted that $r$ may have any positive integral value, but when $r>n$ we have

$$
p_{n+1}=p_{n+2}=\ldots=p_{r}=0 .
$$

To prove Lemma (i), note that

$$
\begin{gathered}
(1-\alpha x)\left(1+\alpha x+\alpha^{2} x^{2} \ldots+\alpha^{m} x^{m}\right)=1-\alpha^{m+1} x^{m+1} \\
\therefore \quad \Pi(1-\alpha x) \cdot \Pi\left(1+\alpha x+\alpha^{2} x^{2}+\ldots+\alpha^{m} x^{m}\right)=\Pi\left(1-\alpha^{m+1} x^{m+1}\right) .
\end{gathered}
$$

Now

$$
\Pi\left(1+\alpha x+\ldots+\alpha^{m} x^{m}\right)=1+\mathbf{H}_{1} x+\mathbf{H}_{2} x^{2}+\ldots+\mathbf{H}_{m} x^{m}+x^{m+1} \mathbf{R}_{m}^{\prime}
$$

where $R_{m}{ }^{\prime} \equiv$ a polynomial in $x$.

And $\Pi\left(1-\alpha^{m+1} x^{m+1}\right)=1+R_{m}{ }^{\prime \prime} x^{m+1}$, where $R_{m}{ }^{\prime \prime} \equiv$ a polynomial in $x$. 
Hence

$$
\begin{aligned}
\Pi(1-\alpha x) \cdot\left\{1+\mathrm{H}_{1} x+\mathrm{H}_{\mathrm{g}} x^{2}+\ldots+\mathrm{H}_{m} x^{m}\right\} & =1+x^{m+1}\left(\mathbf{R}_{m}{ }^{\prime}-\Pi(1-\alpha x) \mathbf{R}_{m}{ }^{\prime \prime}\right) \\
& =1+x^{m+1} \mathbf{R}_{m}
\end{aligned}
$$

where $R$ is a finite polynomial in $x$.

Proof of Lemma (ii):

$$
\text { We have } \begin{aligned}
\frac{\Pi(1-\alpha x)}{1-\alpha x} & \equiv(1-\beta x)(1-\gamma x) \ldots(1-\lambda x) \\
& =1-a_{1} x+a_{2} x^{2}-\ldots+a_{n-1}(-x)^{n-1}
\end{aligned}
$$

where $a_{r} \equiv$ the elementary symmetric function of degree $r$ of the $n-1$ letters $\beta, \gamma, \ldots \lambda$.

Similarly $\frac{\Pi(1-\alpha x)}{(1-\beta x)}=1-b_{1} x+b_{2} x^{2} \ldots+b_{n-1}(-x)^{n-1}$

where $b_{r} \equiv$ the value of $a_{r}$ with $\alpha$ and $\beta$ interchanged, and so on.

Hence $\Sigma \frac{\Pi(1-\alpha x)}{1-\alpha x}=n-x \Sigma a_{1}+x^{2} \Sigma a_{2} \ldots+(-x)^{m-1} \Sigma a_{n-1}$

the summation extending over $n$ terms, one of the letters $\alpha, \beta \ldots \lambda$ being omitted each time.

It is obvious that $\Sigma a_{r}=(n-r) p_{r}$, since any particular combination occurs as often as there are letters not involved in it, viz., $n-r$ times.

Hence

$$
\Sigma \frac{\Pi(1-\alpha x)}{1-\alpha x}=n-(n-1) p_{1} x+(n-2) p_{2} x^{2}-\ldots+p_{n-1}(-x)^{n-1} .
$$

To prove Lemma (iii), consider the expression :

$$
\begin{aligned}
\Sigma\left\{\left(1+2 \alpha x+3 \alpha^{2} x^{2}+\ldots+\right.\right. & \left.\overline{m+1} \alpha^{m} x^{m}\right)\left(1+\beta x+\ldots+\beta^{m} x^{m}\right) \\
& \left.\left(1+\gamma x+\ldots+\gamma^{m} x^{m}\right) \ldots\left(1+\lambda x+\ldots+\lambda^{m} x^{m}\right)\right\}
\end{aligned}
$$

where the summation extends over $n$ terms, in each of which one of the letters $\alpha, \beta, \ldots \lambda$ occurs in a factor similar to that involving $\alpha$ in the typical term attached to the $\Sigma$. If this expression be expanded, we get a set of terms of the type $\mathrm{Ca}^{a} \beta^{b} \ldots \lambda^{b} x^{a+b \ldots+l}$, where $\mathbf{C}$ is a numerical factor and the indices $a, b, \ldots l$ are positive integers or zeros. The terms having the same values of $a, b, \ldots l$ are $n$ in number, and each has one of the values $a+1, b+1, \ldots l+1$ as numerical coefficient. Hence the sum of such terms is

$$
\{(a+1)+(b+1)+\ldots+(l+1)\} \alpha^{a} \beta^{b} \ldots \lambda^{l} x^{a+b \ldots+l}
$$

so that the numerical coefficient $=n+\Sigma a=n+r$ where $r$ is the index of the power of $x$. 
Thus the coefficient of $x^{r}$ in the expansion is

$$
(n+r) \Sigma\left(\alpha^{a} \beta^{b} \gamma^{c} \ldots \lambda^{l}\right),
$$

where $a, b, c \ldots$ take all positive integral or zero values that make $\Sigma a=r$, so that $\Sigma\left(\alpha^{a} \beta^{b} \ldots \lambda^{b}\right)=\mathbf{H}_{r}$, provided $m \nless r$.

Hence we have

$$
\begin{aligned}
& \Sigma\left\{\left(1+2 \alpha x+3 x^{2} x^{2}+\ldots+(m+1) \alpha^{m} x^{m}\right)\left(1+\beta x+\ldots+\beta^{m} x^{m}\right) \ldots\right. \\
&\left.\left(1+\lambda x+\ldots+\lambda^{m} x^{m}\right)\right\} \\
&=n+(n+1) \mathrm{H}_{1} x+(n+2) \mathrm{H}_{2} x^{2}+\ldots+(n+r) \mathrm{H}_{r}+\ldots+(n+m) \mathrm{H}_{m} x^{m}
\end{aligned}
$$

+ a finite polynomial in $x$ having no power of $x$ lower than $x^{m+1}$.

To prove Lemma (iv) :

$$
\begin{gathered}
\left(1+2 \alpha x+3 \alpha^{2} x^{2}+. .+(m+1) \alpha^{m} x^{m}\right)(1-\alpha x) \\
=1+\alpha x+\alpha^{2} x^{2} \ldots+\alpha^{m} x^{m}-\overline{m+1} \alpha^{m+1} x^{m+1} \\
\left(1+\beta x+\beta^{2} x^{2}+\ldots+\beta^{m} x^{m}\right)(1-\beta x)=1-\beta^{m+1} x^{m+1}, \text { etc. }
\end{gathered}
$$

Hence

$$
\begin{gathered}
\begin{array}{c}
\left\{1+2 \alpha x+3 \alpha^{2} x^{2}+\ldots+(m+1) \alpha^{m} x^{m}\right\}\left(1+\beta x+\beta^{2} x^{2}+. .+\beta^{m} x^{m}\right) \ldots \\
\ldots\left(1+\lambda x \ldots+\lambda^{m} x^{m}\right) \Pi(1-\alpha x) \\
=\left\{1+\alpha x+\alpha^{2} x^{2}+\ldots+\alpha^{m} x^{m}-(m+1) \alpha^{m+1} x^{m+1}\right\} \\
\times\left(1-\beta^{m+1} x^{m+1}\right) \ldots\left(1-\lambda^{m+1} x^{m+1}\right)
\end{array} \\
=1+\alpha x+\alpha^{2} x^{2} . .+\alpha^{m} x^{m}+x^{m+1} \times(\text { a finite polynomial in } x) .
\end{gathered}
$$

Summing, over $n$ equations of this type, we have

$$
\begin{gathered}
\Pi(1-\alpha x) \cdot \Sigma\left\{\left(1+2 \alpha x+3 \alpha^{2} x^{2} \ldots+(m+1) \alpha^{m} x^{m}\right)\right. \\
\left.\left(1+\beta x+\beta^{2} x^{2} \ldots+\beta^{m} x^{m}\right) \ldots\left(1+\lambda x \ldots+\lambda^{m} x^{m}\right)\right\} \\
=n+s_{1} x+s_{2} x^{2} \ldots+s_{m} x^{m}+x^{m+1} \times(\text { a finite polynomial in } x) .
\end{gathered}
$$

To prove Formulae I., we have by Lemma (i)

$$
\begin{aligned}
\left\{1-p_{1} x+p_{2} x^{2} \ldots+p_{n}(-x)^{n}\right\}\left\{1+\mathrm{H}_{1} x+\mathrm{H}_{2} x^{2}+\ldots+\mathrm{H}_{m} x^{m}\right\} & =1+x^{m+1} \mathbf{R}_{m} .
\end{aligned}
$$

Equating coefficients of $x, x^{2}, \ldots x^{r}$, we get the formulae as stated above.

To prove Formulae II, we have by Lemmas (iii) and (iv)

$$
\begin{gathered}
\left\{1-p_{1} x+p_{2} x^{2} \ldots+p_{n}^{\lfloor}(-x)^{n}\right\}\left\{n+(n+1) \mathrm{H}_{1} x+\ldots\right. \\
\left.\ldots+(n+2) \mathrm{H}_{2} x^{2}+\ldots+(n+m) \mathrm{H}_{m} x^{m}\right\} \\
=n+s_{1} x+s_{2} x^{2}+\ldots+s_{m} x^{m}+\text { a finite polynomial in } x \text { con- } \\
\text { taining only powers higher than } x^{m} .
\end{gathered}
$$


Hence, equating coefficients of $x^{r}$, $(n+r) \mathrm{H}_{r}-p_{1}(n+r-1) \mathrm{H}_{r-1}+p_{2}(n+r-2) \mathrm{H}_{r-2}-\ldots$

$$
\ldots+(-1)^{r-1} p_{r-1}(n+1) \mathrm{H}_{1}+(-1)^{r} n p_{r}+\varepsilon_{r}
$$

But by I. $\quad(n+r)\left(\mathrm{H}_{r}-p_{1} \mathrm{H}_{r-1}+p_{2} \mathrm{H}_{r-2} \ldots+(-1)^{r} p_{r}\right)=0$.

Subtracting, we get

$\mathrm{H}_{r-1} p_{1}-2 \mathrm{H}_{r-2} p_{2}+3 \mathrm{H}_{r-3} p_{3} \ldots+(-1)^{r}(r-1) \mathrm{H}_{3} p_{r-1}-(-1)^{r} r p_{r}=8_{r}$ which is the type-formula of II.

To prove formulae III, we multiply both sides of the identity in Lemma (iv) by $\left(1+\mathrm{H}_{1} x+\mathrm{H}_{2} x^{2}+\ldots+\mathrm{H}_{m} x^{m}\right)$. and making use of Lemmas (i) and (iii) we get

$$
\begin{gathered}
\left(1+x^{m+1} \mathrm{R}_{m}\right)\left\{n+(n+1) \mathrm{H}_{1} x+(n+2) \mathrm{H}_{2} x^{2}+\ldots\right. \\
\left.\cdots+(n+m) \mathrm{H}_{m} x^{m}+x^{m+1} \mathrm{R}_{m}{ }^{\prime}\right\} \\
=\left(n+s_{1} x+s_{2} x^{2}+\ldots+s_{m} x^{m}+x^{m+1} \mathrm{R}_{m}^{\prime \prime}\right)\left(1+\mathrm{H}_{1} x+\ldots+\mathrm{H}_{m} x^{m}\right) .
\end{gathered}
$$

Equating coefficients of $x^{r}$ we get

$$
(n+r) \mathrm{H}_{r}=n \mathrm{H}_{r}+s_{1} \mathrm{H}_{r-1}+s_{2} \mathrm{H}_{r-2}+\ldots+s_{r-2} \mathrm{H}_{1}+s_{r}
$$

which at once reduces to the typical formula in III. 